the angular velocity of rotation and the mutual velocity, and dependent on the angle between them. Previous heat-conduction measurements in wide tubes had given a third-power mutual friction of the Gorter-Mellink type associated with turbulence in the superfluid, and it was suggested that this turbulence took the form of a confused tangle of vortex lines, mutual friction being due to collisions between normal fluid excitations and the vortex lines. A calculation based on this mechanism leads to a cubic mutual friction of the right order of magnitude with a collision diameter for roton-vortex line collisions of about $10 \mathrm{~A}$., but a number of difficulties arise in attempting to derive a complete theory. It now appears that different results obtained at Yale with a similar experiment can be reconciled with tho present observations.

The theory of vortex lines is again used in explaining the results of experiments by Dr. H. E. Hall (Cambridge), in which measurements are made of the forces producing angular acceleration and retardation of helium II contained between closely spaced disks. For an angular velocity of $1 \mathrm{rad} / \mathrm{sec}$. the amount of angular momentum collected on stopping the rotation has the full classical value, but for smaller angular velocities it falls progressively below this, the missing angular momentum corresponding to a persistent current, which is probably irrotational and has a life. time of more than $0.25 \mathrm{hr}$. The simplest mechanism for the generation of such persistent currents is the removal of vortex lines by the Magnus effect, but the actual mechanism is probably more complicated. Comparison of the frictional forces observed using spacers of different diameters between the disks suggests that at an early stage in the retardation process the superfluid becomes turbulent with an irrotational mean flow. At superfluid velocities less than a critical value turbulence decays on the disk surfaces and retardation stops.

Following the success of this first discussion day, plans are going forward for a second meeting, which will be concerned with low-temperature distillation, and will take place early in the present year.

D. F. BREWER

\section{THE TEACHING OF METEOROLOGY IN SCHOOLS}

$I^{\mathrm{N}}$

the past, the teaching of meteorology in British schools was largely a matter of climates and trade winds, with but little study of the actual weather outside the windows or much relevance to the future lives of most of the pupils. The October 1956 number of the Royal Meteorological Society's magazine Weather, which has the teaching of meteorology as its main theme, gives evidence of the coming of a more vivid, physical and realistic approach.

A group of H.M. Inspectors of Schools contribute the main article, "Meteorology in Secondary Schools", which deals with the reasons for studying physical meteorology as well as geographical climatology in schools and provides an outline course of study. The group points out that the weather may be of vital importance to some pupils after they have left school and will at least be of interest to all. Apart from future use of knowledge of the processes of weather, study of them provides illustrations of important physical principles and, in simple observing work, an introduction to scientific accuracy and method. The outline course opens with the basic physics of radiation, condensation, relation of wind to pressure, etc., and goes on to properties of air masses and fronts and methods of observation. It is stressed that the teacher should correlate his lesson on air-masses by reference to the weather of the day but does not mention the interpretation of the broadcast forecasts. The latter is the most obvious omission in the course and is regrettable because weather forecasts will be used to greater or lesser degree by all the pupils in adult life. An amusing note is provided by the examples of awkward questions which pupils might ask on matters not understandable without more advanced physics or mathematics.

Mr. J. B. Rigg describes the meteorological studies at Watford Grammar School. These begin at the age of 11 years with the making of simple rain-gauges. In the sixth form the geography students, who include many also taking physics and mathematics, carry out much practical work with the comprehensive collection of instruments.

A number of publications, such as the "Observer's Handbook", are mentioned in the articles. Reference might also have been made to the Ministry of Agriculture pamphlet, "Weather and the Land", written by the Agriculture Branch of the Meteorological Office, which gives admirable guidance in the interpretation of the broadcast weather forecasts to meet local conditions.

An editorial discusses whether it is likely to be possible to teach any of the more recently gained knowledge in meteorology in sixth forms. It considers that there is a great opportunity for physics teachers to show the operation of basic physics in the weather, but little possibility of modern dynamical meteorology being taught because in their honours courses most mathematics teachers have not learnt real hydrodynamics with vorticity and viscosity.

\section{UNUSUAL UPPER-AIR REFRACTION PHENOMENON}

$T$ 'HE optical phenomenon of 'looming' which occurs when the air temperature increases very markedly with height is well known at low altitudes, especially in the polar regions. The rays of light from distant objects are then much more strongly curved in an arc convex upwards than usual, so that distant objects are apparently raised and multiple images successively upright and inverted are seen. This is the effect opposite to the mirage associated with a very steep fall of temperature with height, in which the rays become convex downwards and the 'sky is seen on looking along the ground.

The August number of the Meteorological Magazine contains an account by C. S. Durst, G. A. Bull and E. J. Sumner of 'looming' of distant clouds seen from a Canberra aircraft of the Royal Air Force flying north-east at $45,000 \mathrm{ft}$. some 200 miles off the west coast of Norway at lat. $66^{\circ} 20^{\prime} \mathrm{N}$., long. $2^{\circ} 30^{\prime} \mathrm{E}$., at midday on November 29, 1955. The crew, Flying Officers E. E. Kortens and F. P. Fraser, saw a cloud like a bowler hat protrude upwards above the cirrus cloud before and ahead of them. It expanded vertically and sideways. Then another cloud lump appeared at the top of the first one and also spread sideways and vertically. Next a mushroom-like top appeared and finally another lump on top of the 
mushroom. They likened the effect to published photographs of atomic bomb explosions and considered it was larger than any thunder cloud they had ever seen. No estimate of the distance was possible. The colour was white at first, later turning yellow-orange. At this stage they had to turn southwards and could not look backwards.

In turning, they noticed the temperature changed by 6 deg. C. Investigation revealed that an upperair temperature observation at Keflavik, Iceland, at 3 a.m. on the same day included an unusual increase of temperature (inversion) by 4 deg. C. between 43,500 and 45,000 ft., well in the stratosphere, and that the wind distribution was such that this inversion would have reached the position of the aircraft at the time of the observation. Further, cumulonimbus clouds (towering thunder clouds) were observed at stations on the north-west coast of Norway during the morning.

From this it is concluded that the aircraft flew through the inversion and that the abnormal refraction within the inversion produced the distorted vision of these distant clouds. Photographs very similar in appearance of the Bavarian Alps have been taken in known inversion conditions from a peak in the Black Forest. Few similar observations have been reported by aircraft crews, unless some so-called 'flying saucer' reports have such a phenomenon as their basis, and certainly none from so great a height.

The Meteorological Office would welcome additional aircraft reports, especially for the light they would throw on the existence of unusual thermal structure at great heights.

\section{ORDINARY FAMILIES AND THE SOCIAL SERVICES}

$\mathrm{W}$

ITH the financial assistance of the Nuffield Foundation, Political and Economic Planning is undertaking an investigation into the impact of the social services in Britain upon ordinary families. A pilot survey has already been made, and, in view of the importance of the findings, PEP has decided to make these available bofore the main survey is completed*.

The original proposal for the inquiry grew out of the considerable amount of research already carried out by PEP on different aspects of social welfare policy. 'This indicated that the time had come when further assessment of the working of the different services would be helped by a survey aimed at collecting information on the attitude of consumers to the services and on the extent to which their needs are being satisfied or overlooked.

In order to make the group studied reasonably homogeneous, only families with children were included in the survey. The inquiry was made into conditions affecting ordinary families and did not concentrate upon families in special difficulties, even though such families may be heavy claimants on the social services.

The basic purpose of the inquiry was to throw light on the extent to which it is felt that the needs of ordinary families have been met as well as to provide information on the role of the social services in family life. * Planning. Vol. 22, No. 403 (8 October 1956) : Ordinary Families
and the Social Services-Piloting an knquiry. Pp. 189-204. (London : Political and Economic Planning, 1956.) 2s. $6 d$.
An early step in the inquiry was to consult social workers to discuss the occasions when the different social services were likely to be used by ordinary families. Preliminary interviews were then carried out with some families in which discussion was based upon the points which had been formulated by this means ; but no formal questionnaire was used. The interviews were long and all the situations relevant to use of the social services were discussed, ranging from childbirth to care of the aged. These interviews provided case-study material and showed that it was possible to find out the kind of use made of these services by a particular family.

Increased concern for the interests of the family as a whole has been one of the features of social thought in recent years. Attention has been directed to gaps in the working of welfare services and to overlapping or division of function leading to inefficiency in action. In spite of the wide range of statutory services it is still possible for families to get into difficulties which threaten their stability. The PEP inquiry sought to find out whether families had had difficulty in using particular services or were conscious of occasions when they had needed help but had not obtained it.

The extension of the statutory social services in the post-war years has led to some concern lest people should be encouraged to seek help outside the family circle, with a consequent lessening of personal sense of responsibility. It is believed by some that people are now looked after from the cradle to the grave, and that the Welfare State is the final realization of the aims of social reformers. It is also believed that the community has gone too far in taking over responsibility formerly left to families and individuals; that the cost of the social services bears too hard upon certain sections of the population; and that the present system of universal benefits is not needed in conditions of full employment and a high standard of living. The inquiry hoped to find out how far these attitudes are reflected in the opinions of ordinary families.

For the pilot inquiry, the main need was to interview as quickly as possible a large enough number of families to be able to judge whether the information could be obtained and handled as planned. Certain areas were chosen for convenience of interviewing in three different London boroughs, and random calls were made and interviews carried out wherever there was a mother with children in the house available for interview. In all, ninety mothers have been interviewed so far, and two fathers. There have been very few refusals. Interviews all took at least an hour, and sometimes two.

So far the inquiry has been entirely exploratory. Questions have been asked to obtain the following information :

How do mothers regard the help they receive? Do they feel their families are helped a great deal or not very much ? Of which parts of the social services are they most aware? Is there a Welfare State attitude? What form does it take? How does the feeling of different sections vary? Which are the most important services? With which do families and mothers have a lot of contact? What differences are there between different sections of the population in contact with the services? Which services are considered to have worked well ? How do people view the amount of help given? To what extent is there failure to obtain any service needed and how does it arise? What difficulties are experienced ? How have families managed in particular situations of difficulty ? 